\title{
Dynamic analysis of an axially translating viscoelastic beam with an arbitrarily varying length
}

Received: 23 December 2009 / Published online: 6 March 2010

(C) The Author(s) 2010. This article is published with open access at Springerlink.com

\begin{abstract}
The nonlinear free vibration of an axially translating viscoelastic beam with an arbitrarily varying length and axial velocity is investigated. Based on the linear viscoelastic differential constitutive law, the extended Hamilton's principle is utilized to derive the generalized third-order equations of motion for the axially translating viscoelastic Bernoulli-Euler beam. The coupling effects between the axial motion and transverse vibration are assessed under various prescribed time-varying velocity fields. The inertia force arising from the longitudinal acceleration emerges, rendering the coupling terms between the axial beam acceleration and the beam flexure. Semi-analytical solutions for the governing PDE are obtained through the separation of variables and the assumed modes method. The modified Galerkin's method and the fourth-order RungeKutta method are employed to numerically analyze the resulting equations. Further, dynamic stabilization is examined from the system energy standpoint for beam extension and retraction. Extensive numerical simulations are presented to illustrate the influences of varying translating velocities and viscoelastic parameters on the underlying dynamic responses. The material viscosity always dissipates energy and helps stabilize the transverse vibration.
\end{abstract}

\section{Introduction}

The class of axially moving continua has found wide application in diverse mechanical systems such as serpentine belt systems, band saws, high-speed magnetic paper tapes, and power transmission chains, etc. The traveling, tensioned Bernoulli-Euler beam and the traveling flexible string are the most common models of such type of axially moving media. The pioneering work of axially moving continua is ascending to Mote [1] and Ulsoy and Mote [2] who investigated the vibration of a band saw and first introduced the gyroscopic items into axially translating system. Wickert and Mote [3-5] presented the summary work of axially moving continua. Subsequently, Rao [6] studied the free transverse vibration of a traveling string on multiple elastic supports, as well as the active structural control of the string vibration. A beam moving with time-dependent axial velocity was examined by Pakdemirli and Ozkaya [7] based on a systematic approach of Lie group theory. However, in the foregoing studies, the length of the beam is constant, not time-varying, though some considered varying axial moving velocities.

L. H. Wang $\cdot$ Z. D. Hu $\cdot$ Z. Zhong

School of Aerospace Engineering and Applied Mechanics, Tongji University,

Shanghai, People's Republic of China

L. H. Wang · J. W. Ju ( $ه)$

Department of Civil and Environmental Engineering, University of California,

Los Angeles, CA 90095-1593, USA

E-mail: juj@ucla.edu 
With the advancement of high-performance mechanical systems such as robotic manipulators, high-rise elevators, satellite flexible appendages, crane and mining hoists, etc., beam models with a prismatic joint at one end and a moving boundary on the other end have attracted extensive attention. In these beam models, the length of the beam is time-variant, and the system turns into a moving boundary value problem as the spatial domain changes with time. Tabarrok et al. [8] first studied the dynamics of a translating beam with varying length. They derived the equations of motion of a simple cantilever beam model utilizing Newton's second law, and assumed a special velocity profile to obtain a semi-analytic solution for specific axial velocities and approximate solutions for various velocities. A perturbation method was introduced by Zajaczkowski and Lipinski [9] to investigate the parametric instability of the motion of a cantilever beam; however, their model was restricted to the periodically varying length. Wang and Wei [10] studied the vibration problem of a moving slender prismatic beam using a modified Galerkin method with time-dependent basis functions based on Newton's second law. However, their derivation leaves out certain terms resulting in incorrect conclusions with regard to the stability analysis during extension and retraction.

Buffinton [11] applied the assumed mode technique to model the flexible robots containing axially moving elastic members and treated the beam's finite number of supports as kinematical constraints imposed on an unconstrained beam. For earlier work on string with a mass-spring system emulating an elevator, we refer to Yamamoto et al. [12] and Terumichi et al. [13]; both studies assumed a constant transport velocity. Zhu and $\mathrm{Ni}[14]$ presented the linear dynamics of a cantilever beam with an arbitrarily varying length where the tension from their axially moving acceleration was incorporated; they also studied the dynamic stability from the energy viewpoint. Base on the finite element method, Stylianou and Tabarrok $[15,16]$ investigated the axially moving slender beam; their numerical results specified that the beam would be stabilized in extension and unstabilized in retraction. The dynamics and control of a translating flexible beam with a tip mass at one end emerging from or retracting into a rigid base was proposed by Tadikonda and Baruh [17]; they exploited the eigenfunctions of a cantilever beam to obtain closed-form expressions for several domain integrals that arise in the model, which showed that the coupling effect of elastic and translational motions is very important to the beam control. Moreover, using Hamiltonian dynamic analysis, Wang et al. [18] investigated an axially translating elastic Bernoulli-Euler cantilever beam featuring time-variant velocity. Clearly, the stability analysis of dynamical systems is very important; we refer to Zajaczkowski and Lipinski [9], Theodore et al. [19], Pakdemirli and Ulsoy [20], and Wang et al. [18] for further studies.

In the aforementioned literature, the beams were assumed to be linearly elastic, and damping was ignored since the primary focus was on the intrinsic stability. Nevertheless, in the past few decades, there has been considerable progress in material technologies. Many new materials are now commonly adopted for moving continua, such as metal or ceramics reinforced composites (e.g., steel-cord or glass-cord) and polymeric materials (e.g., amorphous polymers, semi-crystalline polymers, and biopolymers). These new materials often do not obey the Hooke's law, but exhibit inherently viscoelastic behavior. Therefore, the application of viscoelasticity attracts substantial attention within the axially moving continuum field. For example, Fung et al. [21] explored the transverse vibrations of an axially moving viscoelastic string subjected to an initial stress on the uniform cross section; they applied the Galerkin's method to solve the equations of motion. The multiple scales method was presented by Yang and Chen [22] for obtaining the near- and exact-resonant steady-state response of the forced vibration of a simply supported axially moving viscoelastic beam. Zhang and Zu [23,24] attempted to describe the mechanical energy dissipation using a viscoelastic model for the belt, and utilized the perturbation techniques to predict the non-linear response. These viscoelastic studies provided a systematic methodology to incorporate material damping in the analysis; however, they did not constitute a significant material dissipation mechanism in the derivations of the equations of motion.

Further, Oz et al. [25] surveyed the nonlinear vibrations and stability of a simply supported beam traveling at harmonically time-varying velocity. Pelicano and Vestroni [26] and Pelicano et al. [27] also investigated bifurcations and parametric resonances of a moving beam; several different viscoelastic models were proposed for different applications, and the key results were verified by experimental measurements. A spectral element model was introduced by Lee and Oh [28] to study the dynamics and stability of an axially moving viscoelastic beam subject to axial tension. Chen and Yang [29] explored the bifurcation and chaos of an axially accelerating viscoelastic beam and introduced the four-term Galerkin truncation to identify dynamical behaviors. Marnowski and Kapitaniak [30] used a three-parameter Zenter element to model the axially moving beam subjected to time-dependent tension; they also investigated the bifurcation and chaos issues. In Hou and Zu's [31] work, for comparing the three representative viscoelastic models to evaluate the viscoelasticity of the axially moving belts, they demonstrated that the standard linear solid (SLS) model containing three elements can be degenerated to the Kelvin model and Maxwell model, which are mostly used for simplicity. The preliminary 
comparisons and numerical simulations showed that the SLS model provided the most accurate solution. In the subcritical and supercritical speed ranges, the vibration characteristics of a light axially moving band was investigated by Koivurova [32] based on the finite element analysis. In the foregoing research on the nonlinear problems of axially moving media, all studies were restricted to the cases with a constant span length; further, most parts of the literature only dealt with a constant transport velocity.

In this paper, we present the dynamic analysis of an axially translating viscoelastic beam with an arbitrarily varying length and a variable transport velocity. Emanating from the linear viscoelastic differential constitutive law and utilizing the standard linear solid (SLS) model, the generalized equations of motion are derived for an axially translating beam with geometric nonlinearities based on Hamilton's principle. The modified Galerkin's method is adopted to truncate the governing equations into a set of nonlinear ordinary differential equations. The fourth-order Runge-Kutta method is employed to solve the governing nonlinear partial differential equations of motion. Since the boundedness of the displacement does not ensure the boundedness of the energy of vibration for a time-varying system [33], the dynamic system stability is carefully examined from the energy standpoint. Extensive numerical examples are also presented to manifest the effects of viscoelastic parameters, axial moving velocities, and the geometric nonlinearity upon the amplitudes of free-vibration responses. Based on the proposed fundamental dynamic analysis, further vibration control can be adopted for such axially moving systems in the near future.

\section{Governing equations of motion}

\subsection{Viscoelastic governing equations}

The SLS model is a popular and versatile viscoelastic material model, more general than the elastic, Kelvin and Maxwell models which are often found inadequate. For instance, the Maxwell model does not account for creep, and the Kelvin-Voigt model does not accommodate stress relaxation. The SLS model is the simplest viscoelastic model that allows for both creep and stress relaxation; it favorably predicts the general shape of the stress-strain responses as well as instantaneous and asymptotic behaviors. Therefore, we adopt the SLS model in this paper to gain a general insight into the effect of material damping on the dynamic response of an axially translating viscoelastic beam.

The SLS model for viscoelasticity is exhibited in Fig. 1, which is a three-parameter model. Specifically, $E_{1}$ and $E_{2}$ are the elastic Young's moduli of the springs; $\eta$ is the dynamic viscosity constant of the dashpot. Further, $\sigma$ is the applied local stress; $\varepsilon_{1}$ and $\varepsilon_{2}$ are the Lagrangian strains. In what follows, a prime or a dot over a variable denotes the partial derivative with respect to the spatial Cartesian coordinate $x$ or time $t$. For a one-dimensional problem, the stress-strain relationship is expressed as

$$
\begin{aligned}
& \left\{\begin{array}{l}
\sigma=E_{1} \varepsilon_{1}, \\
\varepsilon=\varepsilon_{1}+\varepsilon_{2},
\end{array}\right. \\
& \sigma=E_{2} \varepsilon_{2}+\eta \dot{\varepsilon}_{2} .
\end{aligned}
$$

Taking the Laplace transform of Eqs. (1) and (2), we arrive at

$$
\left(E_{2}+s \eta+E_{1}\right) \bar{\sigma}=E_{1}\left(E_{2}+s \eta\right) \bar{\varepsilon} .
$$

The inverse Laplace transform of Eq. (3) then leads to

$$
\begin{aligned}
\left(E_{1}+E_{2}\right) \sigma+\eta \dot{\sigma} & =E_{1} E_{2} \varepsilon+E_{1} \eta \dot{\varepsilon}, \\
\sigma+P_{1} \dot{\sigma} & =Q_{0} \varepsilon+Q_{1} \dot{\varepsilon},
\end{aligned}
$$

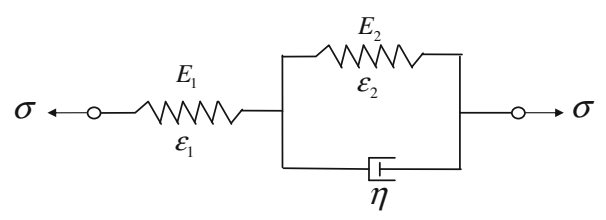

Fig. 1 The standard linear solid model 
where $Q_{1}>P_{1} Q_{0}$ and

$$
P_{1}=\frac{\eta}{E_{1}+E_{2}}, \quad Q_{0}=\frac{E_{1} E_{2}}{E_{1}+E_{2}}, \quad Q_{1}=\frac{E_{1} \eta}{E_{1}+E_{2}}
$$

The bending moment $M$ at the point $x$ and the time $t$ in the vibrating beam reads

$$
M(x, t)=\int_{A}-\sigma(x, y, t) y \mathrm{~d} A .
$$

The Laplace transform of Eq. (7) results in

$$
\bar{M}=\int_{A}-\bar{\sigma} y \mathrm{~d} A .
$$

Substitution of Eq. (3) into Eq. (8) yields

$$
\bar{M}=-\int_{A} \frac{E_{1}\left(E_{2}+s \eta\right)}{\left(E_{2}+s \eta+E_{1}\right)} \bar{\varepsilon} y \mathrm{~d} A .
$$

We can rephrase Eq. (9) as

$$
\left(E_{2}+s \eta+E_{1}\right) \bar{M}=-E_{1}\left(E_{2}+s \eta\right) \int_{A} \bar{\varepsilon} y \mathrm{~d} A .
$$

The inverse Laplace transform of Eq. (10) gives

$$
\left(E_{2}+E_{1}\right) M+\eta \dot{M}=-E_{1} E_{2} \int_{A} \varepsilon y \mathrm{~d} A-E_{1} \eta \int_{A} \dot{\varepsilon} y \mathrm{~d} A .
$$

The flexural strain in the beam takes the form

$$
\varepsilon=-\frac{y}{r(x)}=-y \kappa(x)
$$

where $r(x)$ is the radius of curvature and $\kappa=\frac{1}{r}$ is the longitudinal curvature. By substituting Eq. (12) into Eq. (11) and considering only small deformation with $w^{\prime} \ll 1$, we write

$$
\left(E_{2}+E_{1}\right) M+\eta \dot{M}=-E_{1} E_{2} I w^{\prime \prime}-E_{1} \eta I \dot{w}^{\prime \prime},
$$

where $I=\int_{A} y^{2} \mathrm{~d} A$ is the moment of inertia of the beam cross-section and $w(x, t)$ is the transverse deflection of the beam. Equation (13) can also be recast as

$$
M+P_{1} \dot{M}=S_{0} w^{\prime \prime}+S_{1} \dot{w}^{\prime \prime},
$$

where $P_{1}$ is defined in Eq. (6) and

$$
S_{0}=\frac{E_{1} E_{2} I}{E_{1}+E_{2}}, \quad S_{1}=\frac{E_{1} \eta I}{E_{1}+E_{2}}, \quad S_{1}>P_{1} S_{0} .
$$




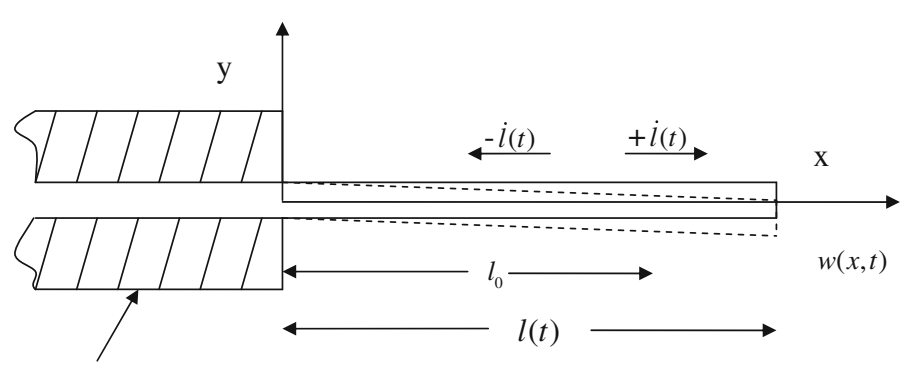

Prismatic joint

Fig. 2 The schematic of an axially translating beam

\subsection{Equations of motion}

The extended Hamilton's principle is used to derive the governing equations of motion. The Bernoulli-Euler beam theory is applied, and the beam deflection is based on the small deflection assumption. As shown in Fig. 2, we consider a uniform viscoelastic cantilever beam which translates at a time-varying axial velocity $\dot{i}(t) ; l(t)$ is the instantaneous length of the beam external to the prismatic joint and is a function of time $t$. A positive or negative transport velocity means that the beam is in extension or retraction mode.

The kinetic energy of the beam is expressed as

$$
T=\frac{1}{2} \int_{0}^{l(t)} \rho\left\{c^{2}+\left(\dot{w}+c w^{\prime}\right)^{2}\right\} \mathrm{d} x=\frac{1}{2} \int_{0}^{l(t)} \rho\left\{c^{2}+\left(\frac{D w}{D t}\right)^{2}\right\} \mathrm{d} x,
$$

where

$$
\frac{D}{D t} \equiv \frac{\partial}{\partial t}+c \frac{\partial}{\partial x}
$$

defines the differentiation with respect to the motion and $\rho$ is the mass per unit length. The potential energy of the beam takes the form

$$
V=\frac{1}{2} \int_{0}^{l(t)}\left[P(x, t) w^{\prime 2}+M(x, t) w^{\prime \prime}\right] \mathrm{d} x,
$$

where $P(x, t)$ signifies the tension in the beam at the position $x$ and reads

$$
P(x, t)=-\rho[l(t)-x] \dot{c} ;
$$

when the beam moves at a uniform axial speed, we have $P(x, t)=0$. The applied work from the external force $p(x, t)$ is

$$
W=\int_{0}^{l(t)} p(x, t) w(x, t) \mathrm{d} x .
$$

In this paper, for simplicity, we consider only the free vibration and therefore $W=0$. When dealing with the problem of forced vibration, the particular solution should be added to the homogeneous solution presented in this paper.

According to the extended Hamilton's principle, we write

$$
\delta \int_{t_{1}}^{t_{2}}(T-V) \mathrm{d} t+\int_{t_{1}}^{t_{2}} \delta W \mathrm{~d} t=0
$$


The Leibnitz integral rule leads to

$$
\frac{\partial}{\partial t} \int_{0}^{l(t)} \rho \frac{D w}{D t} \delta w \mathrm{~d} x=\int_{0}^{l(t)} \rho \frac{\partial}{\partial t}\left(\frac{D w}{D t}\right) \delta w \mathrm{~d} x+\int_{0}^{l(t)} \rho \frac{D w}{D t} \delta \dot{w} \mathrm{~d} x+\left.i(t) \rho \frac{D w}{D t} \delta w\right|_{x=l(t)} .
$$

Therefore, we arrive at

$$
\int_{0}^{l(t)} \rho \frac{D w}{D t} \delta \dot{w} \mathrm{~d} x=\frac{\partial}{\partial t} \int_{0}^{l(t)} \rho \frac{D w}{D t} \delta w \mathrm{~d} x-\left.c \rho \frac{D w}{D t} \delta w\right|_{x=l(t)}-\int_{0}^{l(t)} \rho \frac{\partial}{\partial t}\left(\frac{D w}{D t}\right) \delta w \mathrm{~d} x .
$$

Integrating (23) from $t_{1}$ to $t_{2}$ and imposing vanishing variations of $\delta w$ at $t_{1}$ and $t_{2}$ yields

$$
\int_{t_{1}}^{t_{2}} \int_{0}^{l(t)} \rho \frac{D w}{D t} \delta \dot{w} \mathrm{~d} x \mathrm{~d} t=-\left.\int_{t_{1}}^{t_{2}} \rho c\left(\frac{D w}{D t} \delta w\right)\right|_{x=l(t)} \mathrm{d} t-\int_{t_{1}}^{t_{2}} \int_{0}^{l(t)} \rho \frac{\partial}{\partial t}\left(\frac{D w}{D t}\right) \delta w \mathrm{~d} x \mathrm{~d} t .
$$

Substituting Eqs. (16), (18) and (20) into (21) and invoking Eq. (24), we obtain

$$
\int_{t_{1}}^{t_{2}} \int_{0}^{l(t)}\left[\rho \frac{D^{2} w}{D t^{2}}-\left(P w^{\prime}\right)^{\prime}+M^{\prime \prime}\right] \delta w \mathrm{~d} x+\left.\int_{t_{1}}^{t_{2}} P w^{\prime} \delta w\right|_{x=0} ^{x=l(t)} \mathrm{d} t+\left.\int_{t_{1}}^{t_{2}} \rho c \frac{D w}{D t} \delta w\right|_{x=0} \mathrm{~d} t=0
$$

where

$$
\frac{D^{2} w}{D t^{2}}=\ddot{w}+2 c \dot{w}^{\prime}+\dot{c} w^{\prime}+c^{2} w^{\prime \prime}
$$

Since $\delta w$ is arbitrary, the equation of motion emerges as

$$
\rho\left(\ddot{w}+2 c \dot{w}^{\prime}+\dot{c} w^{\prime}+c^{2} w^{\prime \prime}\right)-\left[P(x, t) w^{\prime}\right]^{\prime}+M^{\prime \prime}=0 \quad \text { for } 0<x<l(t) .
$$

The first four terms in Eq. (27) correspond to the local, Coriolis, tangential, and centripetal acceleration, respectively. The fifth term defines the coupling between the axial beam acceleration and the beam curvature, thus vanishing if the axial motion occurs at a constant velocity (i.e., $\ddot{l}(t)=0$ ). If the beam's axial velocity is zero, the second to fourth terms in Eq. (27) would vanish. Further, if there is no viscosity $\eta$, we recover the elastic flexure $M=E I w^{\prime \prime}$; as a result, only the first and the last terms in Eq. (27) would remain, which represents the transverse vibration of a non-translating beam.

Multiplying Eq. (27) by the operator $\left(1+P_{1} \frac{\partial}{\partial t}\right)$ and Eq. (14) by the operator $\frac{\partial^{2}}{\partial x^{2}}$, and substituting the modified Eq. (14) into the modified Eq. (27), we arrive at

$$
\left(1+P_{1} \frac{\partial}{\partial t}\right)\left\{\rho\left(\ddot{w}+2 c \dot{w}^{\prime}+\dot{c} w^{\prime}+c^{2} w^{\prime \prime}\right)-\left[P(x, t) w^{\prime}\right]^{\prime}\right\}+\left(S_{0}+S_{1} \frac{\partial}{\partial t}\right) w^{\prime \prime \prime \prime}=0, \quad 0<x<l(t) .
$$

The boundary conditions of a cantilever beam are

$$
\begin{array}{ll}
\text { At } x=0: & w(0, t)=w^{\prime}(0, t)=0, \\
\text { At } x=l(t): & S_{0} w^{\prime \prime}+S_{1} \dot{w}^{\prime \prime}=S_{0} w^{\prime \prime \prime}+S_{1} \dot{w}^{\prime \prime \prime}=0 .
\end{array}
$$




\section{Analytical solutions of the equations of motion}

The assumed modes method and the Bernoulli-Euler beam theory are adopted here. Moreover, the modified Galerkin's method is employed to discretize the spatial domain of the governing partial differential equations of the viscoelastic beam. By utilizing the classical method of separation of variables, under the boundary conditions (29) and (30), the analytical solutions of Eq. (28) can be expanded into the form

$$
w(x, t)=\sum_{i=1}^{\infty} q_{i}(t) \varphi_{i}(x, t) \approx \frac{1}{\sqrt{l(t)}} \sum_{i=1}^{n} q_{i}(t) \phi_{i}\left(\frac{x}{l(t)}\right),
$$

where $q_{i}(t)$ are the non-dimensional temporal functions, $n$ is the number of included modes, and $\phi_{i}\left(\frac{x}{l}\right)$ is the $i$ th trial function which is also termed the $i$ th assumed mode. The orthonormal eigenfunctions of a cantilever beam are applied as the trial functions, which can be expressed as $\phi_{i}(\xi)=\varphi_{i}(x, t) \sqrt{l(t)}(i=1,2,3 \ldots)$, where $\xi=\frac{x}{l(t)}$.

Since the spatial domain is time-variant, both $\phi_{i}$ and its corresponding eigenvalue $\lambda_{i}$ are also time-variant. An explicit expression for $\phi_{i}(\xi)$ is rendered as (cf. [10])

$$
\phi_{i}(\xi)=\Upsilon_{i}(t)\left\{a_{i}^{-1}\left[\cosh \left(\kappa_{i} \pi \xi\right)-\cos \left(\kappa_{i} \pi \xi\right)\right]-b_{i}^{-1}\left[\sinh \left(\kappa_{i} \pi \xi\right)-\sin \left(\kappa_{i} \pi \xi\right)\right]\right\}, \quad 0 \leq \xi \leq 1,
$$

and

$$
a_{i}=\cosh \left(\kappa_{i} \pi\right)+\cos \left(\kappa_{i} \pi\right), \quad b_{i}=\sinh \left(\kappa_{i} \pi\right)+\sin \left(\kappa_{i} \pi\right),
$$

where $\kappa_{i}$ is the $i$ th root of the transcendental equation

$$
\cos (\kappa \pi)=-1 / \cosh (\kappa \pi) .
$$

Moreover, the normalizing coefficient $\Upsilon_{i}(t)$ reads

$$
\begin{aligned}
\Upsilon_{i}(t)= & \sqrt{\kappa_{i} \pi}\left\{\left(a_{i}^{-2}+b_{i}^{-2}\right)\left[\frac{1}{4} \sinh \left(2 \kappa_{i} \pi\right)-\cosh \left(\kappa_{i} \pi\right) \sin \left(\kappa_{i} \pi\right)\right]\right. \\
& \left.+\left(a_{i}^{-2}-b_{i}^{-2}\right)\left[\frac{1}{4} \sin \left(2 \kappa_{i} \pi\right)-\sinh \left(\kappa_{i} \pi\right) \cos \left(\kappa_{i} \pi\right)\right]+\kappa_{i} \pi a_{i}^{-2}\right] \\
& \left.\left.+2\left(a_{i} b_{i}\right)^{-1}\left[\frac{1}{4}\left(\cos \left(2 \kappa_{i} \pi\right)-\cosh \left(2 \kappa_{i} \pi\right)\right)+\sin \left(\kappa_{i} \pi\right) \sinh \left(\kappa_{i} \pi\right)\right]\right]\right\}^{-1 / 2} .
\end{aligned}
$$

Note that $\kappa_{i}$ is independent of the time $t$. The first three roots of Eq. (34) are $\kappa_{1}=0.597, \kappa_{2}=1.494$ and $\kappa_{3}=2.5$. For $i>3$, we have $\kappa_{i} \approx\left(i-\frac{1}{2}\right)$. By designating $\frac{1}{\sqrt{l}} \phi_{j}\left(\frac{x}{l}\right)$ as the $j$ th weighting function $(j=1, \ldots, n)$, the modified Galerkin's method then yields

$$
\int_{0}^{l(t)} \Pi[w] \frac{1}{\sqrt{l(t)}} \phi_{j}\left(\frac{x}{l(t)}\right)=0,
$$

where $\Pi(w)$ denotes the left-hand side of Eq. (28):

$$
\Pi(w)=\left(1+P_{1} \frac{\partial}{\partial t}\right)\left\{\rho\left(\ddot{w}+2 c \dot{w}^{\prime}+\dot{c} w^{\prime}+c^{2} w^{\prime \prime}\right)-\left[P(x, t) w^{\prime}\right]^{\prime}\right\}+\left(S_{0}+S_{1} \frac{\partial}{\partial t}\right) w^{\prime \prime \prime \prime} .
$$

Equations (31), (36) and (37) then result in the $n$-term Galerkin approximation of the governing viscoelastic equations of motion:

$$
\mathbf{I}(t) \dddot{\mathbf{q}}(t)+\mathbf{M}(t) \ddot{\mathbf{q}}(t)+\mathbf{C}(t) \dot{\mathbf{q}}(t)+\mathbf{K}(t) \mathbf{q}(t)=0,
$$

where $\mathbf{q}=\left[q_{1}, q_{2}, \ldots q_{n}\right]^{T}$ signifies the vector of generalized coordinates. The first term of Eq. (38) involves the third-order derivative of $\mathbf{q}$, which does not exist in the dynamic analysis of an axially translating elastic beam (cf. [18]). This third-order derivative term ascends to Eq. (14) as a direct consequence of using the SLS 
model which gives rise to a higher-order time derivative of the temporal coordinates. For an elastic beam, there will be no such third-order time derivative in the equations of motion. Furthermore, if one adopts the Kelvin viscoelastic model (cf., [22-24,28,29]), there will be no higher-order time derivative for the stress; therefore, the third-order time derivative for the equations of motion will also disappear. Though Fung et al. [21] and Hou and $\mathrm{Zu}$ [31] employed the SLS model, Fung et al. [21] assumed the viscosity constant to be zero for simplicity, and Hou and $\mathrm{Zu}$ [31] applied the multiple scale method in a different fashion. Here, we define I in Eq. (38) as the viscosity matrix:

$$
I_{i j}=P_{1} \delta_{i j}
$$

If the viscosity parameter $\eta$ is negligibly small, I matrix will approach the null matrix, and the viscoelastic beam vibration analysis will be recovered as an elastic case. Moreover, $\mathbf{M}$ is the consistent mass matrix of the beam; $\mathbf{C}$ and $\mathbf{K}$ correspond to the equivalent damping matrix and the equivalent stiffness matrix, respectively. $\mathbf{C}$ matrix is generally non-symmetric since it consists of the non-symmetric matrix from viscoelastic damping and the skew-symmetric matrix from Coriolis acceleration. Likewise, $\mathbf{K}$ matrix is generally non-symmetric as it involves the symmetric matrix from flexural rigidity of the beam, the skew-symmetric matrix from centripetal acceleration, and the non-symmetric matrix from viscoelasticity. In Eq. (38), we write

$$
\begin{aligned}
& M_{i j}=\left[1-\frac{3 P_{1} i(t)}{2 l(t)}\right] \delta_{i j}+2 P_{1} \frac{i(t)}{l(t)} \int_{0}^{1}\left(1-\frac{3}{2} \xi\right) \phi_{i}^{\prime}(\xi) \phi_{j}(\xi) \mathrm{d} \xi, \\
& C_{i j}=\left\{\frac{9}{4} P_{1}\left[\frac{\dot{l}(t)}{l(t)}\right]^{2}-\frac{3}{2} P_{1} \frac{\ddot{l}(t)}{l(t)}-\frac{i(t)}{l(t)}\right\} \delta_{i j}+\left\{9 P_{1}\left[\frac{\dot{l}(t)}{l(t)}\right]^{2}-3 P_{1} \frac{\ddot{l}(t)}{l(t)}-2 \frac{\dot{l}(t)}{l(t)}\right\} \int_{0}^{1} \xi \phi_{i}^{\prime}(\xi) \phi_{j}(\xi) \mathrm{d} \xi \\
& +\left\{2 \frac{i(t)}{l(t)}+2 P_{1} \frac{\ddot{l}(t)}{l(t)}-6 P_{1}\left[\frac{i(t)}{l(t)}\right]^{2}\right\} \int_{0}^{1} \phi_{i}^{\prime}(\xi) \phi_{j}(\xi) \mathrm{d} \xi+3 P_{1}\left[\frac{i(t)}{l(t)}\right]^{2} \int_{0}^{1} \xi^{2} \phi_{i}^{\prime \prime}(\xi) \phi_{j}(\xi) \mathrm{d} \xi \\
& -\left\{4 P_{1}\left[\frac{\dot{l}(t)}{l(t)}\right]^{2}+P_{1} \frac{i(t)}{l(t)}\right\} \int_{0}^{1} \xi \phi_{i}^{\prime \prime}(\xi) \phi_{j}(\xi) \mathrm{d} \xi+\left\{P_{1}\left[\frac{\dot{l}(t)}{l(t)}\right]^{2}+P_{1} \frac{i(t)}{l(t)}\right\} \int_{0}^{1} \phi_{i}^{\prime \prime}(\xi) \phi_{j}(\xi) \mathrm{d} \xi \\
& +\frac{S_{1}}{\rho[l(t)]^{4}} \int_{0}^{1} \phi_{i}^{\prime \prime}(\xi) \phi_{j}^{\prime \prime}(\xi) \mathrm{d} \xi \\
& K_{i j}=\left\{-\frac{15}{8} P_{1}\left[\frac{\dot{l}(t)}{l(t)}\right]^{3}+\frac{9}{4} P_{1} \frac{i(t) \ddot{l}(t)}{[l(t)]^{2}}-\frac{P_{1}}{2} \frac{\dddot{l}(t)}{l(t)}+\frac{3}{4}\left[\frac{\dot{l}(t)}{l(t)}\right]^{2}-\frac{1}{2} \frac{\dddot{l}(t)}{l(t)}\right\} \delta_{i j} \\
& +\left\{-\frac{45}{4} P_{1}\left[\frac{i(t)}{l(t)}\right]^{3}+9 P_{1} \frac{i(t) \ddot{l}(t)}{[l(t)]^{2}}-P_{1} \frac{\dddot{l}(t)}{l(t)}+3\left[\frac{\dot{l}(t)}{l(t)}\right]^{2}-\frac{\ddot{l}(t)}{l(t)}\right\} \int_{0}^{1} \xi \phi_{i}^{\prime}(\xi) \phi_{j}(\xi) \mathrm{d} \xi \\
& +\left\{\frac{15}{2} P_{1}\left[\frac{\dot{l}(t)}{l(t)}\right]^{3}-6 P_{1} \frac{\dot{l}(t) \ddot{l}(t)}{[l(t)]^{2}}-3\left[\frac{\dot{l}(t)}{l(t)}\right]^{2}\right\} \int_{0}^{1} \phi_{i}^{\prime}(\xi) \phi_{j}(\xi) \mathrm{d} \xi \\
& +\left\{-\frac{15}{2} P_{1}\left[\frac{\dot{l}(t)}{l(t)}\right]^{3}+3 P_{1} \frac{i(t) \ddot{l}(t)}{[l(t)]^{2}}+\left[\frac{\dot{l}(t)}{l(t)}\right]^{2}\right\} \int_{0}^{1} \xi^{2} \phi_{i}^{\prime \prime}(\xi) \phi_{j}(\xi) \mathrm{d} \xi \\
& +\left\{10 P_{1}\left[\frac{\dot{l}(t)}{l(t)}\right]^{3}-4 P_{1} \frac{\dot{l}(t) \ddot{l}(t)}{[l(t)]^{2}}-2\left[\frac{\dot{l}(t)}{l(t)}\right]^{2}+\frac{5}{2} P_{1}\left[\frac{\dot{l}(t)}{l(t)}\right]^{2}\right\} \int_{0}^{1} \xi \phi_{i}^{\prime \prime}(\xi) \phi_{j}(\xi) \mathrm{d} \xi,
\end{aligned}
$$




$$
\begin{aligned}
& -\frac{5}{2} P\left\{1\left[\frac{i(t)}{l(t)}\right]^{3}+\left[\frac{i(t)}{l(t)}\right]^{2}\right\} \int_{0}^{1} \phi_{i}^{\prime \prime}(\xi) \phi_{j}(\xi) \mathrm{d} \xi \\
& +P_{1}\left[\frac{i(t)}{l(t)}\right]^{3} \int_{0}^{1} \xi^{3} \phi_{i}^{\prime \prime}(\xi) \phi_{j}^{\prime}(\xi) \mathrm{d} \xi-P_{1}\left\{2\left[\frac{i(t)}{l(t)}\right]^{3}+\left[\frac{i(t)}{l(t)}\right]^{2}\right\} \int_{0}^{1} \xi^{2} \phi_{i}^{\prime \prime}(\xi) \phi_{j}^{\prime}(\xi) \mathrm{d} \xi \\
& +P_{1}\left\{\left[\frac{i(t)}{l(t)}\right]^{3}+\left[\frac{i(t)}{l(t)}\right]^{2}\right\} \int_{0}^{1} \xi \phi_{i}^{\prime \prime}(\xi) \phi_{j}^{\prime}(\xi) \mathrm{d} \xi+\left\{\frac{S_{0}}{\rho[l(t)]^{4}}-\frac{9 S_{1} i(t)}{2 \rho[l(t)]^{5}}\right\} \int_{0}^{1} \phi_{i}^{\prime \prime}(\xi) \phi_{j}^{\prime \prime}(\xi) \mathrm{d} \xi \\
& -\frac{S_{1} i(t)}{\rho[l(t)]^{5}} \int_{0}^{1} \xi \phi_{i}^{\prime \prime \prime}(\xi) \phi_{j}^{\prime \prime}(\xi) \mathrm{d} \xi .
\end{aligned}
$$

On the other hand, the initial conditions $q_{i}(0), \dot{q}_{i}(0)$ and $\ddot{q}_{i}(0)$ can be obtained from: At $t=0$

$$
w(x, 0)=w_{0}(x), \quad \dot{w}(x, 0)=\left.\dot{w}\right|_{t=0}=\dot{w}_{0}(x), \quad \ddot{w}(x, 0)=\left.\ddot{w}\right|_{t=0}=\ddot{w}_{0}(x),
$$

or

$$
\begin{aligned}
w(x, 0)= & \frac{1}{\sqrt{l(0)}} \sum_{i=1}^{n} q_{i}(0) \phi_{i}\left(\frac{x}{l(0)}\right), \\
\dot{w}_{0}(x, 0)= & -\frac{1}{2}[l(0)]^{-\frac{3}{2}} \dot{l}(0) \sum_{i=1}^{n} q_{i}(0) \phi_{i}\left(\frac{x}{l(0)}\right)+\frac{1}{\sqrt{l(0)}} \sum_{i=1}^{n} \dot{q}_{i}(0) \phi_{i}\left(\frac{x}{l(0)}\right) \\
& -x[l(0)]^{-\frac{5}{2}} \dot{l}(0) \sum_{i=1}^{n} q_{i}(0) \phi_{i}^{\prime}\left(\frac{x}{l(0)}\right), \\
\ddot{w}(x, 0)= & \frac{1}{\sqrt{l(0)}} \sum_{i=1}^{n} \ddot{q}_{i}(0) \phi_{i}\left(\frac{x}{l(0)}\right)-[l(0)]^{-\frac{3}{2}} \dot{l}(0) \sum_{i=1}^{n} \dot{q}_{i}(0) \phi_{i}\left(\frac{x}{l(0)}\right) \\
& -2 x[l(0)]^{-\frac{5}{2}} \dot{l}(0) \sum_{i=1}^{n} \dot{q}_{i}(0) \phi_{i}^{\prime}\left(\frac{x}{l(0)}\right) \\
& +\left(\frac{3}{4}[l(0)]^{-\frac{5}{2}}[\dot{i}(0)]^{2}-\frac{1}{2}[l(0)]^{-\frac{3}{2}} \ddot{l}(0)\right) \sum_{i=1}^{n} q_{i}(0) \phi_{i}\left(\frac{x}{l(0)}\right) \\
& +\left(3 x[l(0)]^{-\frac{7}{2}}[i(0)]^{2}-x[l(0)]^{-\frac{5}{2}} \ddot{l}(0)\right) \sum_{i=1}^{n} q_{i}(0) \phi_{i}^{\prime}\left(\frac{x}{l(0)}\right) \\
& +x^{2}[l(0)]^{-\frac{9}{2}}[\dot{l}(0)]^{2} \sum_{i=1}^{n} q_{i}(0) \phi_{i}^{\prime \prime}\left(\frac{x}{l(0)}\right) .
\end{aligned}
$$

Multiplying Eq. (44)-(46) by $\frac{\phi_{j}(x / l(0))}{\sqrt{l(0)}}$ and integrating them from $x=0$ to $l(0)$, we obtain

$$
\begin{aligned}
& q_{i}(0)=\sqrt{l(0)} \int_{0}^{1} w(\xi l(0), 0) \phi_{i}(\xi) \mathrm{d} \xi \\
& \dot{q}_{i}(0)=\sqrt{l(0)} \int_{0}^{1} \dot{w}(\xi l(0), 0) \phi_{i}(\xi) \mathrm{d} \xi+\frac{c(0)}{l(0)} \sum_{j=1}^{n} q_{j}(0) \int_{0}^{1} \xi \phi_{j}^{\prime}(\xi) \phi_{i}(\xi) \mathrm{d} \xi+\frac{c(0)}{2 l(0)} q_{i}(0),
\end{aligned}
$$




$$
\begin{aligned}
\ddot{q}_{i}(0)= & \sqrt{l(0)} \int_{0}^{1} \ddot{w}(\xi l(0), 0) \phi_{i}(\xi) \mathrm{d} \xi+\frac{\dot{l}(0)}{l(0)} \dot{q}_{i}(0)+2 \frac{\dot{l}(0)}{l(0)} \sum_{j=1}^{n} \dot{q}_{j}(0) \int_{0}^{1} \xi \phi_{j}^{\prime}(\xi) \phi_{i}(\xi) \mathrm{d} \xi \\
& +\left(\frac{1}{2} \frac{\ddot{l}(0)}{l(0)}-\frac{3}{4}\left[\frac{\ddot{l}(0)}{l(0)}\right]^{2}\right) q_{i}(0)+\left(\frac{\ddot{l}(0)}{l(0)}-3\left[\frac{\ddot{l}(0)}{l(0)}\right]^{2}\right) \sum_{j=1}^{n} q_{j}(0) \int_{0}^{1} \xi \phi_{j}^{\prime}(\xi) \phi_{i}(\xi) \mathrm{d} \xi \\
& -\left[\frac{\dot{l}(0)}{l(0)}\right]^{2} \sum_{j=1}^{n} q_{j}(0) \int_{0}^{1} \xi^{2} \phi_{j}^{\prime \prime}(\xi) \phi_{i}(\xi) \mathrm{d} \xi,
\end{aligned}
$$

Here, we have the initial beam velocity $c(0)=i(0)$, and the deformation of the cantilever beam can be expressed as

$$
\begin{aligned}
& w(x, 0)=w(l(0), 0)\left[\frac{3}{2}\left(\frac{x}{l(0)}\right)^{2}-\frac{1}{2}\left(\frac{x}{l(0)}\right)^{3}\right], \\
& \dot{w}(x, 0)=\dot{w}(l(0), 0)\left[\frac{3}{2}\left(\frac{x}{l(0)}\right)^{2}-\frac{1}{2}\left(\frac{x}{l(0)}\right)^{3}\right], \\
& \ddot{w}(x, 0)=\ddot{w}(l(0), 0)\left[\frac{3}{2}\left(\frac{x}{l(0)}\right)^{2}-\frac{1}{2}\left(\frac{x}{l(0)}\right)^{3}\right] .
\end{aligned}
$$

\section{Analysis of dynamic stabilization}

The varying length and velocity bring in changes in the amplitude of the transverse beam vibration, which manifests the variation in energy. In order to investigate the stabilization of an axially translating and transversely vibrating beam, we need to account for the changes in total energy consisting of the kinetic energy and the potential energy of the transverse vibration; the kinetic energy of the axial motion is not considered here. The total transverse vibration energy $\left(E_{\mathrm{V}}\right)$ of the protruded part of an extruding or retracting beam takes the form

$$
E_{\mathrm{V}}=\frac{1}{2} \int_{0}^{l(t)}\left\{\rho\left(\dot{w}+c w^{\prime}\right)^{2}+P(x, t) w^{\prime 2}+M(x, t) w^{\prime \prime}\right\} \mathrm{d} x,
$$

where $M(x, t)$ and $P(x, t)$ have been previously defined in Eqs. (14) and (19), respectively. Following the same discretization scheme for $w(x, t), M(x, t)$ can be expressed as

$$
M(x, t)=\sum_{i=1}^{\infty} r_{i}(t) \varphi_{i}(x, t) \approx \frac{1}{\sqrt{l(t)}} \sum_{i=1}^{n} r_{i}(t) \phi_{i}\left(\frac{x}{l(t)}\right) .
$$

Substitution of Eq. (54) into Eq. (14) then yields

$$
\begin{aligned}
& \frac{1}{\sqrt{l(t)}} \sum_{i=1}^{n} r_{i}(t) \phi_{i}\left(\frac{x}{l(t)}\right)+\frac{P_{1}}{\sqrt{l(t)}} \sum_{i=1}^{n} \dot{r}_{i}(t) \phi_{i}\left(\frac{x}{l(t)}\right) \\
& =\frac{S_{0}}{\sqrt{l(t)}} \frac{1}{[l(t)]^{2}} \sum_{i=1}^{n} q_{i}(t) \phi_{i}^{\prime \prime}\left(\frac{x}{l(t)}\right)+\frac{S_{1}}{\sqrt{l(t)}} \frac{1}{[l(t)]^{2}} \sum_{i=1}^{n} \dot{q}_{i}(t) \phi_{i}^{\prime \prime}\left(\frac{x}{l(t)}\right) .
\end{aligned}
$$

Multiplying Eq. (55) by $\frac{1}{\sqrt{l(t)}} \phi_{j}\left(\frac{x}{l(t)}\right)$ and integrating it from $x=0$ to $l(t)$, we arrive at

$$
r_{i}(t)+P_{1} \dot{r}_{i}(t)=\frac{S_{0}}{[l(t)]^{2}} \sum_{j=1}^{n} q_{j}(t) \int_{0}^{1} \phi_{i}(\xi) \phi_{j}^{\prime \prime}(\xi) \mathrm{d} \xi+\frac{S_{1}}{[l(t)]^{2}} \sum_{j=1}^{n} \dot{q}_{j}(t) \int_{0}^{1} \phi_{i}(\xi) \phi_{j}^{\prime \prime}(\xi) \mathrm{d} \xi .
$$


At $t=0$, we write

$$
\begin{aligned}
M(x, 0)= & \frac{1}{\sqrt{l(0)}} \sum_{i=1}^{n} r_{i}(0) \phi_{i}\left(\frac{x}{l(0)}\right) \\
\dot{M}_{0}(x, 0)= & -\frac{1}{2}[l(0)]^{-\frac{3}{2}} \dot{l}(0) \sum_{i=1}^{n} r_{i}(0) \phi_{i}\left(\frac{x}{l(0)}\right)+\frac{1}{\sqrt{l(0)}} \sum_{i=1}^{n} \dot{r}_{i}(0) \phi_{i}\left(\frac{x}{l(0)}\right) \\
& -x[l(0)]^{-\frac{5}{2}} \dot{i}(0) \sum_{i=1}^{n} r_{i}(0) \phi_{i}^{\prime}\left(\frac{x}{l(0)}\right) .
\end{aligned}
$$

Multiplying Eqs. (57) and (58) by $\frac{\phi_{j}(x / l(0))}{\sqrt{l(0)}}$ and integrating the expression from $x=0$ to $l(0)$, we obtain

$$
\begin{aligned}
& r_{i}(0)=\sqrt{l(0)} \int_{0}^{1} M(\xi l(0), 0) \phi_{i}(\xi) \mathrm{d} \xi \\
& \dot{r}_{i}(0)=\sqrt{l(0)} \int_{0}^{1} \dot{M}(\xi l(0), 0) \phi_{i}(\xi) \mathrm{d} \xi+\frac{i(0)}{l(0)} \sum_{i=1}^{n} r_{i}(0) \int_{0}^{1} \xi \phi_{i}^{\prime}(\xi) \phi_{j}(\xi) \mathrm{d} \xi+\frac{i(0)}{2 l(0)} r_{i}(0),
\end{aligned}
$$

where

$$
\begin{aligned}
& M(\xi l(0), 0)=M(x, 0)=w(l(0), 0) \frac{3 S_{0}}{[l(0)]^{2}}\left(1-\frac{x}{l(0)}\right), \\
& \dot{M}(\xi l(0), 0)=\dot{M}(x, 0)=\dot{w}(l(0), 0) \frac{3 S_{1}}{P_{1}[l(0)]^{2}}\left(1-\frac{x}{l(0)}\right) .
\end{aligned}
$$

Using the boundary conditions Eqs. (59) and (60), the fourth-order Runge-Kutta method can be applied to solve Eq. (56). Further, after $q_{i}(t)$ and $r_{i}(t)$ are solved, Eq. (53) can be recast as

$$
\begin{aligned}
E_{\mathrm{V}}= & \frac{\rho}{2} \int_{0}^{1}\left[\sum_{i=1}^{n} \dot{q}_{i}(t) \phi_{i}(\xi)+\frac{\dot{l}(t)}{l(t)} \sum_{i=1}^{n} q_{i}(t) \phi_{i}^{\prime}(\xi)\right]^{2} \mathrm{~d} \xi-\frac{\rho \ddot{l}(t)}{2 l(t)} \int_{0}^{1}(1-\xi)\left[\sum_{i=1}^{n} q_{i}(t) \phi_{i}^{\prime}(\xi)\right]^{2} \mathrm{~d} \xi \\
& +\frac{1}{2[l(t)]^{2}} \int_{0}^{1}\left[\sum_{j=1}^{n} r_{j}(t) \phi_{j}(\xi) \sum_{i=1}^{n} q_{i}(t) \phi_{i}^{\prime \prime}(\xi)\right] \mathrm{d} \xi .
\end{aligned}
$$

If the time derivative $\mathrm{d} E_{\mathrm{v}} / \mathrm{d} t<0$, then the total transverse vibration energy of the beam reduces, thus leading to a stabilized transverse dynamic response. The reduction of transverse vibration energy translates into the increase in kinetic energy of the axial beam movement. By contrast, if the time derivative $\mathrm{d} E_{\mathrm{v}} / \mathrm{d} t>0$, the total transverse vibration energy of the beam increases, therefore resulting in an unstabilized transverse dynamic response. The increase of transverse vibration energy comes from the reduction in kinetic energy of the axial beam movement.

The constant velocity examples are considered here to evaluate the dynamic stabilization of Eq. (63). The resulting total transverse vibration energies of the beam under different scenarios are displayed in Figs. 3, 4, 5, and 6. In particular, Figs. 3 and 4 illustrate that an axially moving beam without viscosity dissipates energy during extension (thus stabilized) but gains energy during retraction (thus unstabilized), which have been confirmed in Stylianou and Tabarrok [15,16] and Theodore et al. [19]. During the beam extension in Fig. 3, the energy of the beam system transfers from the transverse vibration to the axial motion by bringing some mass into the domain of effective length. On the other hand, during the beam retraction in Fig. 4, the energy transfers from the axial motion to the transverse vibration by leaving some mass out of the domain of effective length. We now focus on dynamic vibrations of viscoelastic beams. As the material viscosity always dissipates energy, the total transverse vibration energy decreases considerably faster during the (stabilized) beam extension in Fig. 5, compared with the purely elastic beam in Fig. 3. Moreover, the total transverse vibration energy increases significantly slower during the (unstabilized) beam retraction in Fig. 6, compared with the purely elastic beam in Fig. 4. 


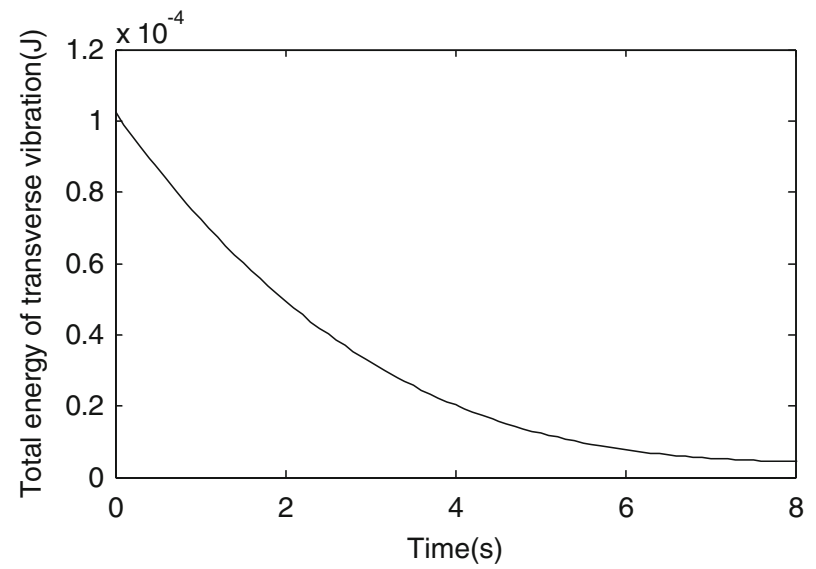

Fig. 3 The transverse vibration energy under the beam extension mode without viscosity when $l_{0}=5.0, i_{(}(t)=1.0$

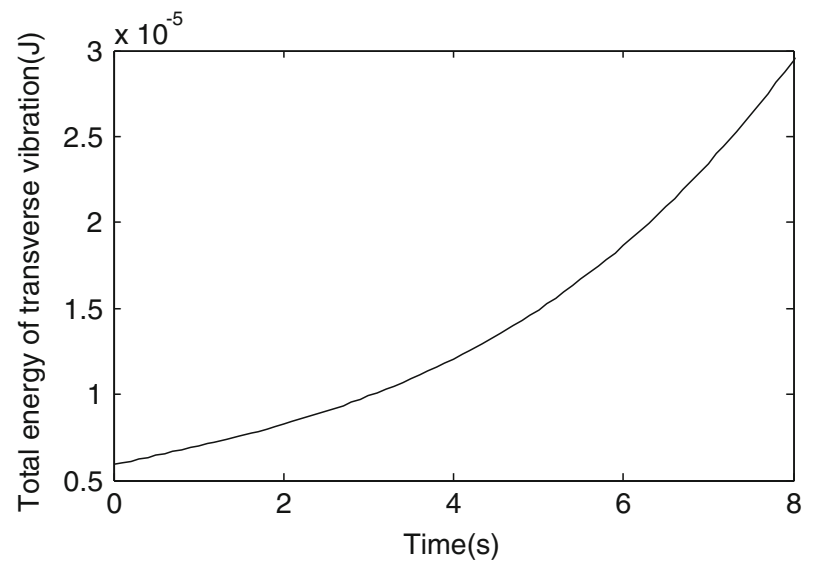

Fig. 4 The transverse vibration energy under the beam retraction mode without viscosity when $l_{0}=15.0, i(t)=-1.0$

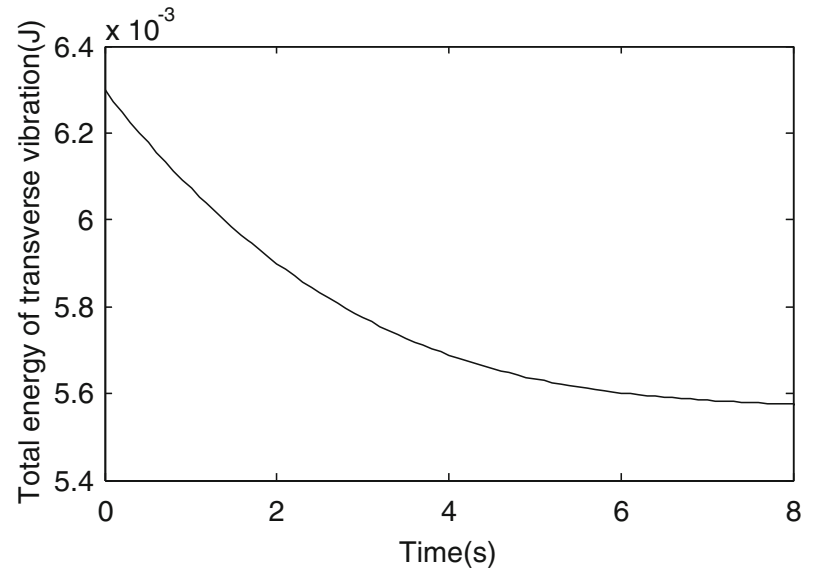

Fig. 5 The transverse vibration energy under the viscoelastic beam extension mode with $\eta=5 \times 10^{-4} E_{2}, l_{0}=5.0, \dot{l}(t)=1.0$

\section{Numerical examples and discussion}

The fourth-order Runge-Kutta method is employed to investigate the effects of viscosity and axial velocity on the transverse vibration and dynamic stabilization of an axially translating viscoelastic beam. The modes of the four lowest orders are utilized here in connection with the assumed modes method; a good fidelity is 


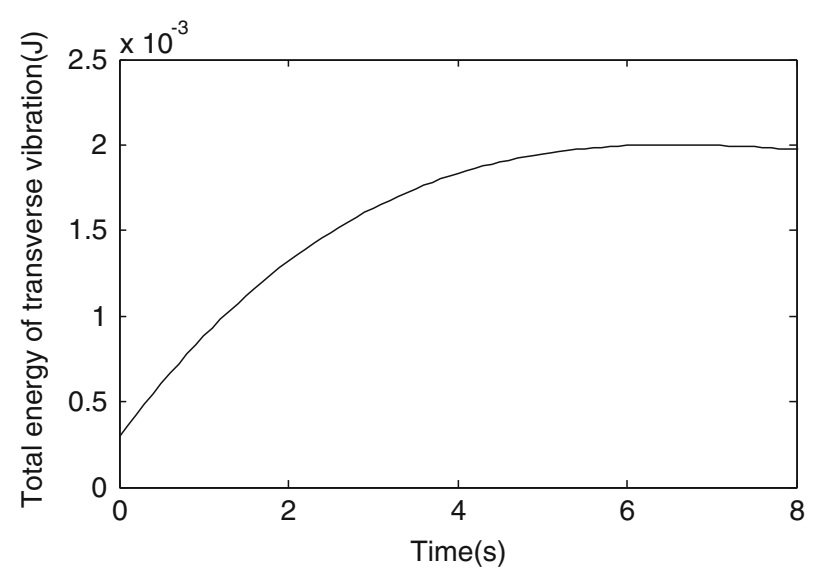

Fig. 6 The transverse vibration energy under the viscoelastic beam retraction mode with $\eta=5 \times 10^{-4} E_{2}, l_{0}=15.0, \dot{l}(t)=-1.0$

achieved. The tip of the cantilever beam is selected to study the vibration of the beam. The effects of varying axial velocities and viscosity coefficients upon the vibration responses are examined in detail.

A prismatic homogeneous steel cantilever beam is considered as an example. The width of the beam is $b=0.2 \mathrm{~m}$; the thickness is $h=0.1 \mathrm{~m}$; the density is $7.8 \times 10^{3} \mathrm{~kg} / \mathrm{m}^{3}$. Further, the stiffness constants of the springs are $E_{1}=2.0 \times 10^{11} \mathrm{~N} / \mathrm{m}^{2}$ and $E_{2}=1.0 \times 10^{11} \mathrm{~N} / \mathrm{m}^{2}$. The initial length of the beam reads $l_{0}$; the initial axial beam velocity is $c_{0}$; the initial translating acceleration is $a_{0}$. The initial conditions of the beam are $w(l(0), 0)=0.001 \mathrm{~m}$ and $\dot{w}(l(0), 0)=0 \mathrm{~m} / \mathrm{s}$.

\subsection{Constant axial translating velocity}

In this special class of numerical simulations, the beam travels at a constant axial velocity. The beam length is expressed as

$$
l(t)=l_{0}+c t
$$

where $l_{0}$ is the initial beam length and $c$ denotes the constant velocity. Different viscosity parameters and axial velocities are compared to manifest their influences on the transient amplitudes of transverse vibrations. In Fig. 7, the tip-deflection time histories are displayed for different dynamic viscosities without axial velocity. Figure 7 shows that the dynamic viscosity takes effect on the vibration amplitude, but it does not affect the vibration frequency; the response amplitude decreases as the dynamic viscosity increases. Figure 8 displays the extending beam vibration amplitudes at a constant velocity with different viscosities; higher viscosity leads to amplitude reduction. By comparison, Fig. 9 illustrates the retracting beam vibration amplitudes at a constant velocity featuring distinct viscosities. From Figs. 7, 8, and 9, we observe that viscoelastic damping substantially affects the dynamic behavior of the beam system. The reduced response amplitude with damping indicates that the effect of viscosity makes the dynamic system more stable.

Further, Figs. 10 and 11 show, respectively, the extruding and retracting beam vibration amplitudes at the tip under different constant velocities. From Fig. 10, we observe that as the beam extends, higher axial translating velocities lead to higher beam tip vibration amplitudes yet lower vibration frequencies. By contrast, from Fig. 11, as the beam retracts, higher absolute axial translating velocities result in lower beam tip vibration amplitudes yet higher vibration frequencies. This is due to the fact that the flexural rigidity of the beam is reduced during the extension mode and enhanced during the retraction mode; i.e., the beam becomes somewhat "softer" and "stiffer", respectively, during the extrusion and retraction operations (cf. [17]).

\subsection{Varying axial translating velocity}

We now consider the transverse vibrations of an axially accelerating or decelerating viscoelastic beam under either extension or retraction operation. 


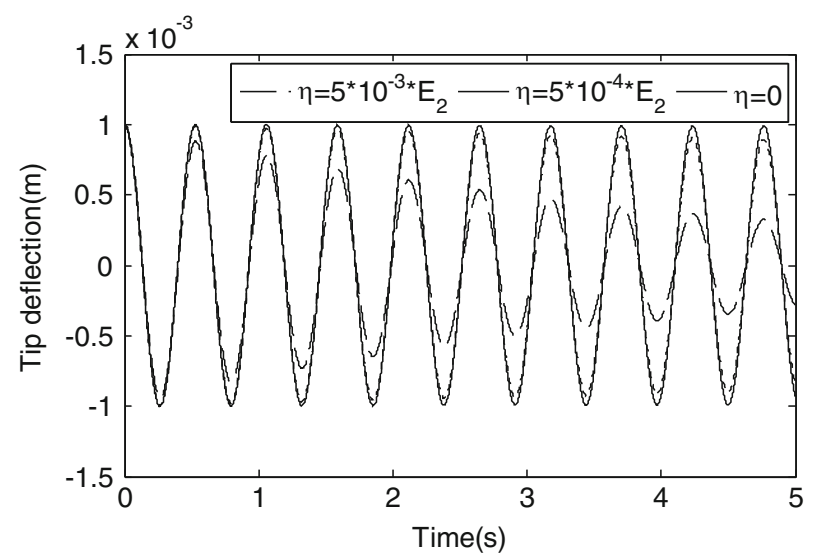

Fig. 7 The influences of various dynamic viscosities on the transient vibration amplitudes of the axially translating cantilever beam with $l_{0}=5.0, i(t)=0$

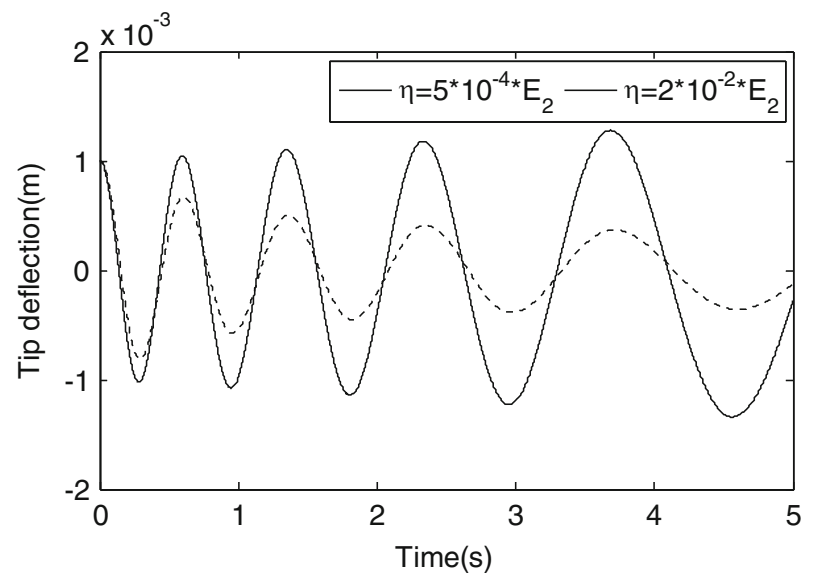

Fig. 8 The extending beam vibration amplitudes at a constant velocity with $l_{0}=5.0, \dot{l}(t)=1.0, \ddot{l}(t)=0$

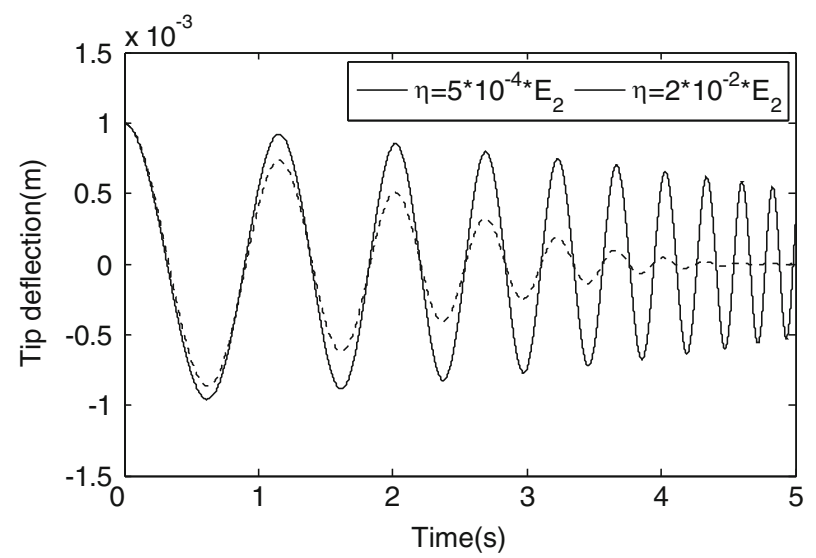

Fig. 9 The retracting beam vibration amplitudes at a constant velocity with $l_{0}=8.0, \dot{l}(t)=-1.0, \ddot{l}(t)=0$

\subsubsection{Case I: Constant axial acceleration}

When the beam travels at a constant acceleration, the beam length is prescribed by

$$
l(t)=l_{0}+c_{0} t+a t^{2} / 2,
$$




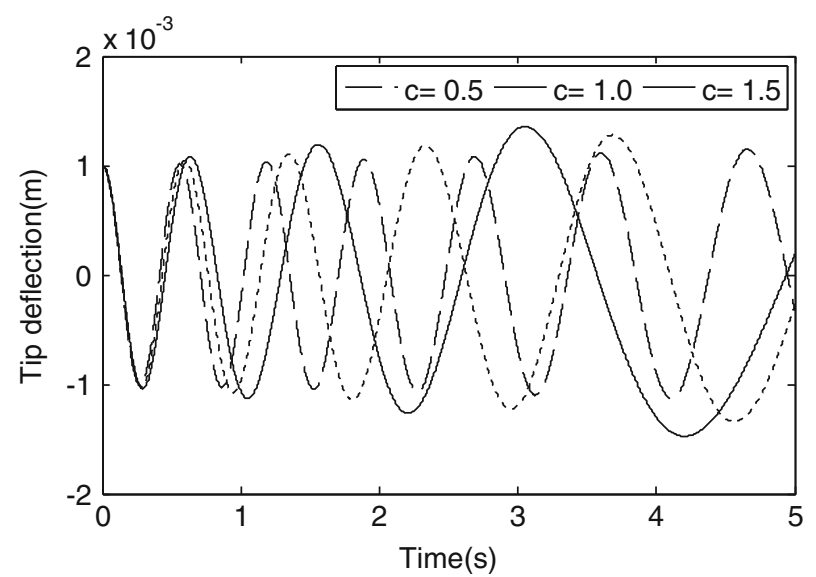

Fig. 10 The extruding beam vibration amplitudes at different constant velocities with $l_{0}=5.0, \eta=5 \times 10^{-4} E_{2}, \ddot{l}(t)=0$

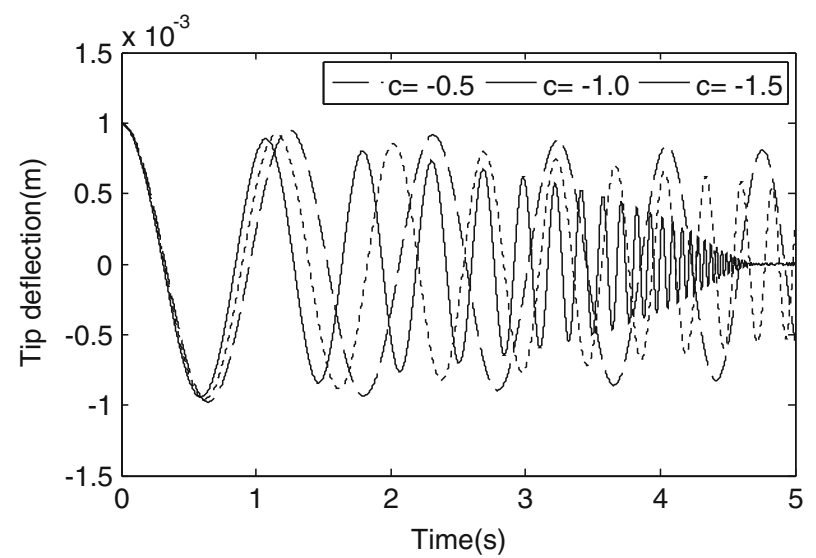

Fig. 11 The retracting beam vibration amplitudes at different constant velocities with $l_{0}=8.0, \eta=5 \times 10^{-4} E_{2}, \ddot{l}(t)=0$

where $c_{0}$ is the initial velocity and $a$ is the constant acceleration. Figures 12 and 13 exhibit the beam tip vibration amplitudes under extension and retraction, respectively, with a constant acceleration (deceleration). Figure 12 shows gradually increasing tip vibration amplitude with decreasing frequency, whereas Fig. 13 displays gradually decreasing tip vibration amplitude with increasing frequency. Figure 14 illustrates the beam tip vibration first under extension then under retraction at a constant acceleration, whereas Fig. 15 renders the reverse sequence of retraction followed by extension at a constant deceleration. In Fig. 14, as the beam vibrates across the first part of the time span under extension, the dynamic system experiences negative damping; as the beam traverses the second part of the time span under retraction, the system then experiences positive damping. By contrast, Fig. 15 illustrates that the damping is positive in the first part of the beam vibration under retraction, and becomes negative during the second part of the time history under extension. Figures 12 , 13,14 , and 15 show that the coupling of axial motion and transverse oscillation is present.

\subsubsection{Case II: Harmonic length variation}

In the following numerical simulations, the length of the beam is characterized by a simple harmonic variation and takes the form

$$
l(t)=l_{0}+\beta \sin (\omega t),
$$

where $\beta$ defines a length variation parameter and $\omega$ signifies the angular frequency of length variation. As illustrated in Figs. 16, 17, 18, and 19, higher angular frequency $\omega$ excites higher vibration modes in the governing equations of motion in Sect. 3; therefore, the contributions from higher modes dominate the beam vibration under harmonic length variation if a higher angular frequency $\omega$ is involved. By comparing 


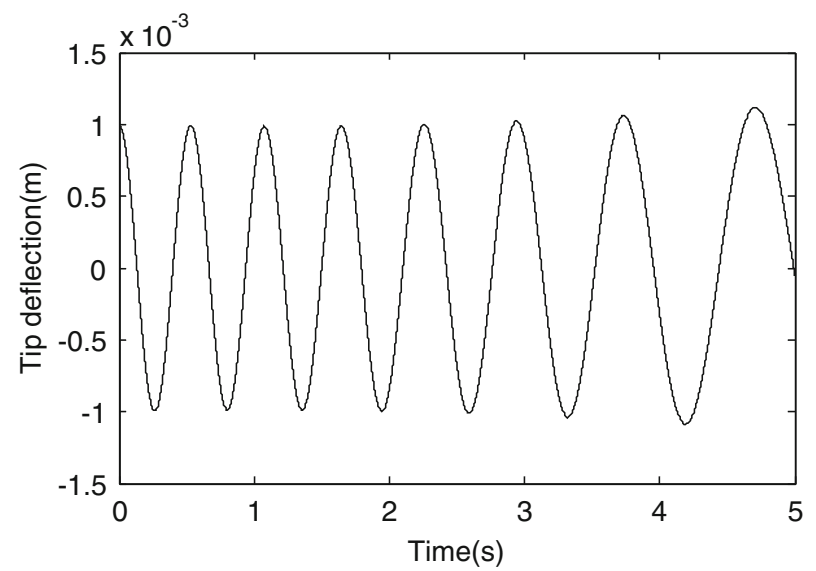

Fig. 12 The extending beam vibration amplitude at a constant acceleration with $l_{0}=5.0, \eta=5 \times 10^{-4} E_{2}, \dot{l}(0)=0, \ddot{l}(t)=0.2$

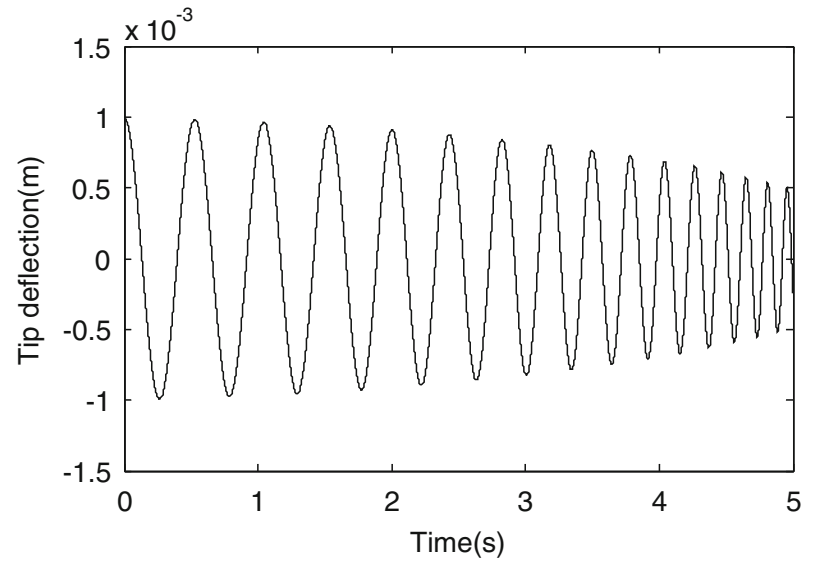

Fig. 13 The retracting beam vibration amplitude at a constant acceleration with $l_{0}=5.0, \eta=5 \times 10^{-4} E_{2}, \dot{l}(0)=0, \ddot{l}(t)=-0.2$

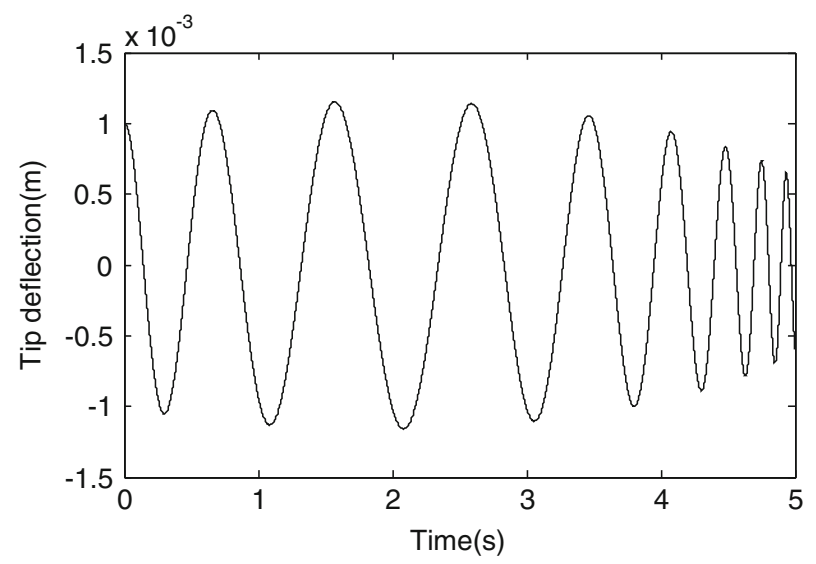

Fig. 14 The extending and retracting beam vibration amplitude at a constant acceleration with $l_{0}=5.0, \eta=5 \times 10^{-4} E_{2}, i(0)=$ 2.0, $\ddot{l}(t)=-1.0$

the amplitude decreases in Figs. 16, 17, 18, and 19 under the same $\beta$ value (0.001), we observe that a higher angular frequency $\omega$ leads to a faster reduction in the vibration amplitude. Therefore, we can reduce and control the transverse vibration amplitude of an axially translating beam through the selection of a suitable angular frequency $\omega$ under the harmonic length variation. Case II presents a better vibration control scheme than Case I. 


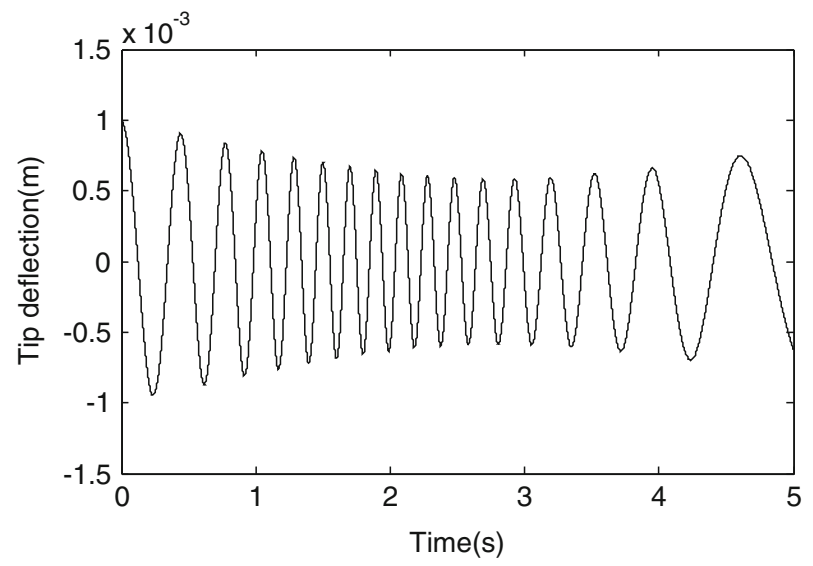

Fig. 15 The retracting and extending beam vibration amplitude at a constant acceleration with $l_{0}=5.0, \eta=5 \times 10^{-4} E_{2}, i(0)=$ $-2.0, \ddot{l}(t)=1.0$

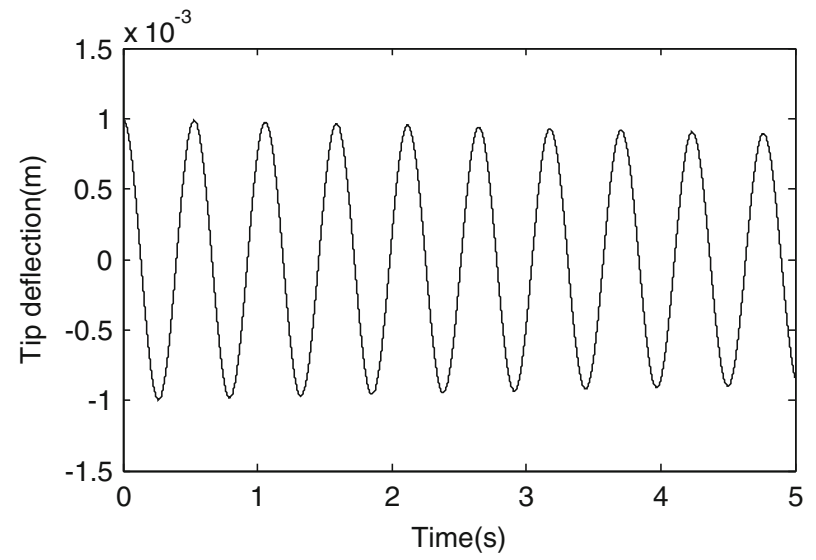

Fig. 16 The beam tip deflection of the harmonic length variation with $l_{0}=5.0, \eta=5 \times 10^{-4} E_{2}, \beta=0.001, \omega=0$ (fixed beam length $l_{0}$ )

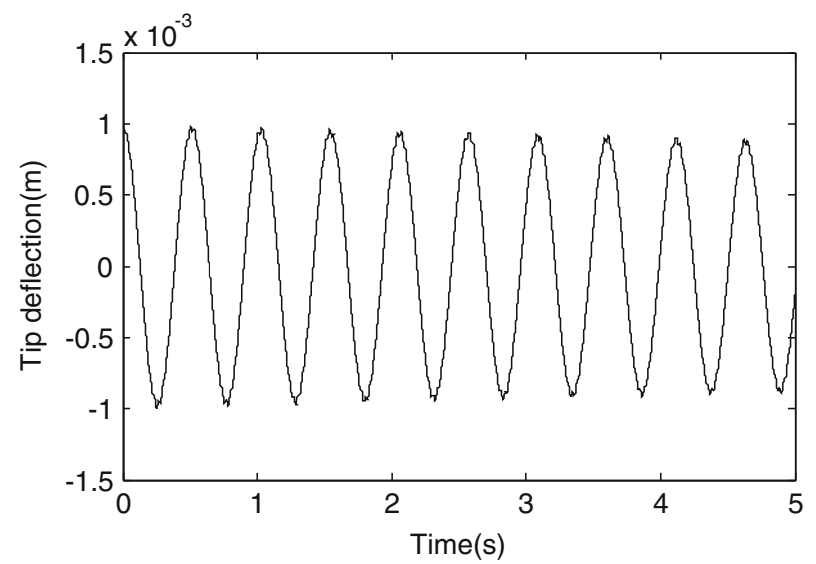

Fig. 17 The beam tip deflection of the harmonic length variation with $l_{0}=5.0, \eta=5 \times 10^{-4} E_{2}, \beta=0.001, \omega=200$

\subsubsection{Case III: Parabolic length variation}

In these numerical simulations, the length of the beam is prescribed by a parabolic variation

$$
l(t)=l_{0}+\gamma \sqrt{t},
$$




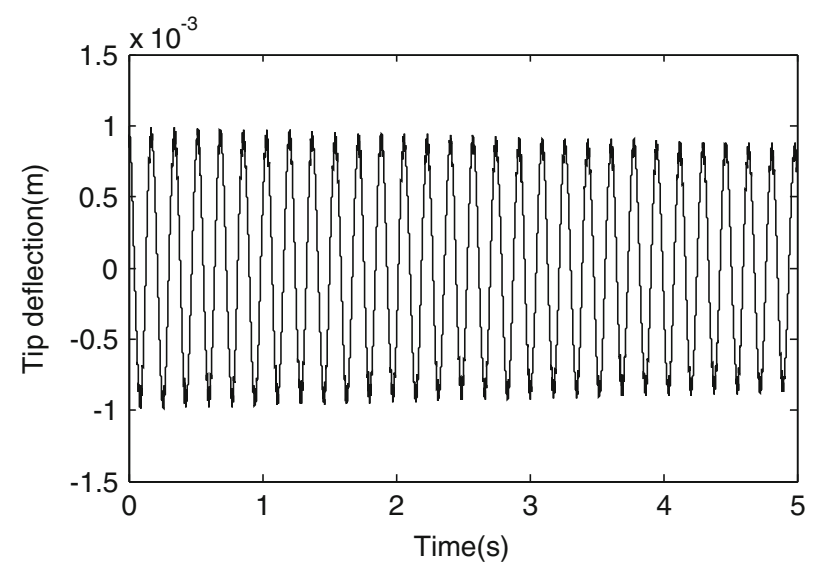

Fig. 18 The beam tip deflection of the harmonic length variation with $l_{0}=5.0, \eta=5 \times 10^{-4} E_{2}, \beta=0.001, \omega=700$

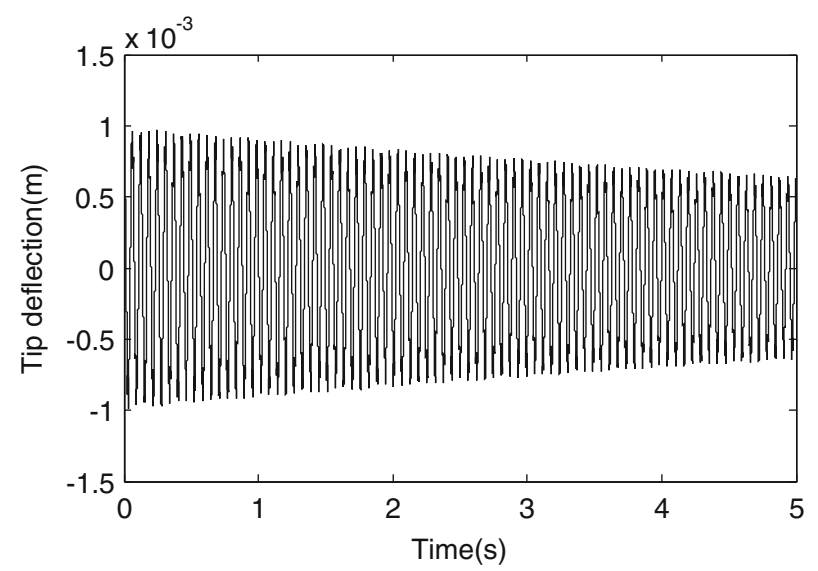

Fig. 19 The beam tip deflection of the harmonic length variation with $l_{0}=5.0, \eta=5 \times 10^{-4} E_{2}, \beta=0.001, \omega=1,200$

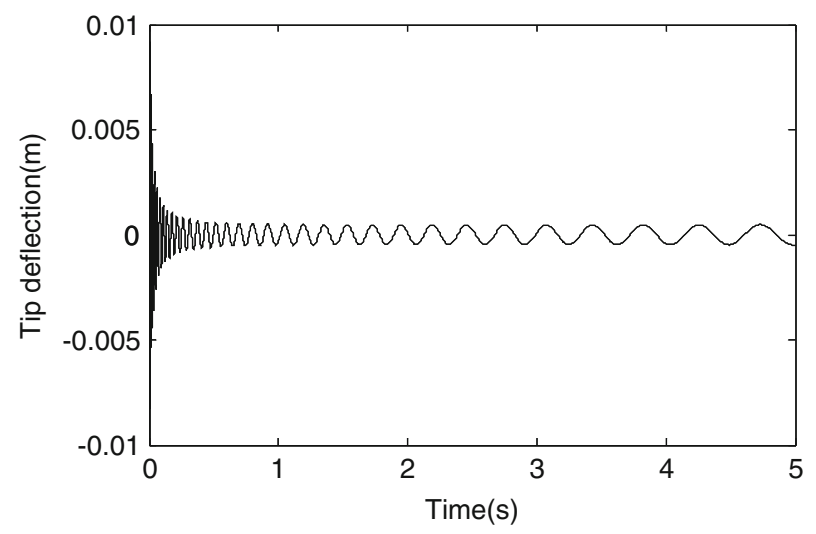

Fig. 20 The beam tip deflection of the parabolic length variation during the extension with $l_{0}=0.5, \eta=5 \times 10^{-4} E_{2}, \gamma=2.0$

where $\gamma$ defines a length variation parameter. Figures 20,21, and 22 display the beam tip deflection time histories of the parabolic length variation under extension or retraction modes with distinct initial beam lengths. Further, in Fig. 20, the initial beam length is relatively short; therefore, during the extension operation, the beam tip deflection amplitude decreases rapidly first and then increases since an increasing beam length results in a reduced flexural stiffness. 


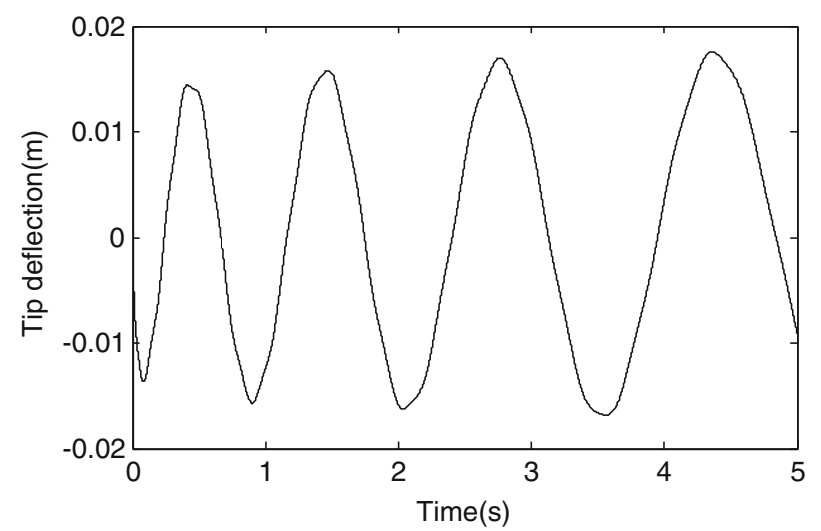

Fig. 21 The beam tip deflection of the parabolic length variation during the extension with $l_{0}=5.0, \eta=5 \times 10^{-4} E_{2}, \gamma=2.0$

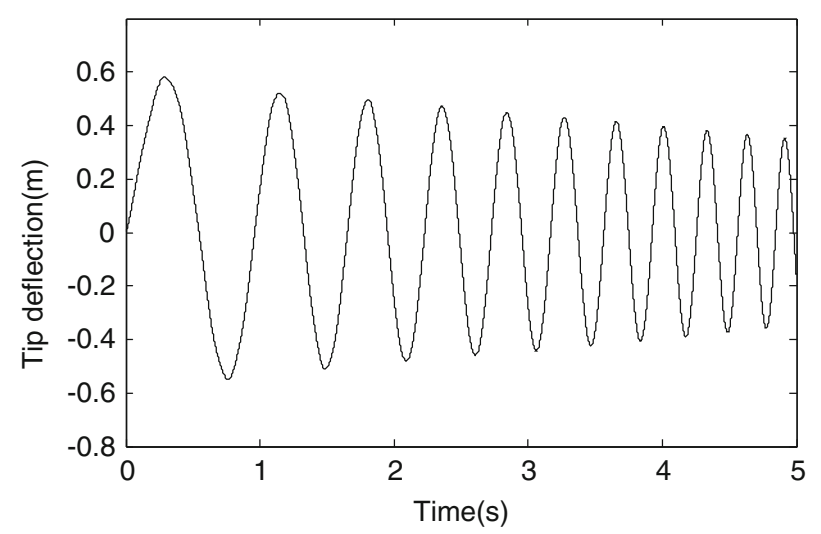

Fig. 22 The beam tip deflection of the parabolic length variation during the retraction with $l_{0}=8.0, \eta=5 \times 10^{-4} E_{2}, \gamma=-2.0$

\section{Conclusion}

In this paper, the extended Hamilton's principle is employed to derive the third-order governing equations of motion under free vibration for an axially moving viscoelastic beam with arbitrarily varying length and axial velocity. The axially translating beam is modeled as a Bernoulli-Euler beam; the viscoelasticity of the beam is represented by the SLS. The coupling effects of axial motion and flexural vibration for a viscoelastic beam are systematically explored. The separation of variables and the assumed mode method are applied to solve the governing partial differential equations. The damping matrix $\mathbf{C}$ is generally non-symmetric as it involves both the viscoelastic damping and Coriolis acceleration. The modified Galerkin's method and the fourth-order Runge-Kutta scheme are utilized to implement extensive numerical simulations. Various motion profiles under constant and varying axial translating velocities are considered to assess the viscoelasticity effects upon flexural vibrations and stabilization for axially moving viscoelastic beams. The numerical results indicate that during the beam extension higher axial translation velocity leads to higher beam tip vibration amplitude yet lower vibration frequency. By contrast, during the beam retraction, higher absolute axial translation velocity results in lower beam tip vibration amplitude yet higher vibration frequency. The stabilization analysis shows that energy transfers from the transverse vibration energy to the axial motion during the beam extension mode, whereas energy transfers from the axial motion to the transverse vibration during the beam retraction mode. The material viscosity always dissipates energy and helps stabilize the transverse vibration of an axially moving viscoelastic beam system in both extension and retraction modes. The present dynamic analysis can facilitate further development in dynamic control of axially moving systems in practice. 
Open Access This article is distributed under the terms of the Creative Commons Attribution Noncommercial License which permits any noncommercial use, distribution, and reproduction in any medium, provided the original author(s) and source are credited.

\section{References}

1. Mote, C.D. Jr.: Dynamic stability of axially moving materials. Shock Vib. Dig. 4, 2-11 (1972)

2. Ulsoy, A.G., Mote, C.D. Jr.: Band saw vibration and stability. Shock Vib. Dig. 10, 3-15 (1978)

3. Wickert, J.A., Mote, C.D. Jr.: Current research on the vibration and stability of axially moving materials. Shock Vib. Dig. 20, 3-13 (1988)

4. Wickert, J.A., Mote, C.D. Jr.: On the energetics of axially moving continua. J. Acoust. Soc. Am. 85, 1365-1368 (1989)

5. Wickert, J.A., Mote, C.D. Jr..: Classical vibration analysis of axially moving continua. ASME J. Appl. Mech. 57, 738-744 (1990)

6. Rao, G.V.: Linear dynamics and active control of an elastically supported traveling string. Comput. Struct. 43, 1041-1049 (1992)

7. Pakdemirli, M., Ozkaya, E.: Group-theoretic approach to axially accelerating beam problem. Acta Mech. 155, $111-123(2002)$

8. Tabarrok, B., Leech, C.M., Kim, Y.I.: On the dynamics of an axially moving beam. J. Frankl. Inst. 297, 201-220 (1974)

9. Zajaczkowski, J., Lipinski, J.: Instability of the motion of a beam of periodically varying length. J. Sound Vib. 63, 9-18 (1979)

10. Wang, P.K.C., Wei, J.: Vibration in a moving flexible robot arm. J. Sound Vib. 116, 149-160 (1987)

11. Buffinton, K.W.: Dynamics of elastic manipulators with prismatic joints. ASME J. Dyn. Syst. Meas. Control 113, 3440 (1992)

12. Yamamoto, T., Yasuda, K., Koto, M.: Vibration of a string with time-variable length. Bull. Jpn. Soc. Mech. Eng. 21, 16771684 (1978)

13. Terumichi, Y., Ohtsuka, M., Yoshizawa, M., Fukawa, Y., Tsujioka, Y.: Nonstationary vibrations of a string with time-varying length and a mass-spring system attached at the lower end. Nonlinear Dyn. 12, 39-55 (1997)

14. Zhu, W.D., Ni, J.: Energetic and stability of translating media with an arbitrarily varying length. J. Vib. Acoust. 122, 295$304(2000)$

15. Stylianou, M., Tabarrok, B.: Finite element analysis of an axially moving beam. Part I: time integration. J. Sound Vib. 178, 433-453 (1994)

16. Stylianou, M., Tabarrok, B.: Finite element analysis of an axially moving beam. Part II: stability analysis. J. Sound Vib. 178, 455-481 (1994)

17. Tadikonda, S.S.K., Baruh, H.: Dynamics and control of a translating flexible beam with a prismatic joint. ASME J. Dyn. Syst. Meas. Control 114, 422-427 (1992)

18. Wang, L.H., Hu, Z.D., Zhong, Z., Ju, J.W.: Hamiltonian dynamic analysis of an axially translating beam featuring time-variant velocity. Acta Mech. 206, 149-161 (2009)

19. Theodore, R.J., Arakeri, J.H., Ghosal, A.: The modeling of axially translating flexible beams. J. Sound Vib. 191, $363-$ 376 (1996)

20. Pakdemirli, M., Ulsoy, A.G.: Stability analysis of an axially accelerating string. J. Sound Vib. 203, 815-832 (1997)

21. Fung, R.F., Huang, J.S., Chen, Y.C., Yao, C.M.: Nonlinear dynamics analysis of the viscoelastic string with a harmonically varying transport speed. Comput. Struct. 66, 777-784 (1998)

22. Yang, X.D., Chen, L.Q.: Nonlinear forced vibration of axially moving viscoelastic beams. Acta Mech. Solida Sin. 19, 365373 (2006)

23. Zhang, L.X., Zu, J.W.: Nonlinear vibrations of viscoelastic moving belts. Part I: free vibration analysis. J. Sound Vib. 216, 75$91(1998)$

24. Zhang, L.X., Zu, J.W.: Nonlinear vibrations of viscoelastic moving belts. Part II: forced vibration analysis. J. Sound Vib. 216, 93-105 (1998)

25. Oz, H.R., Pakdemirli, M., Boyaci, H.: Non-linear vibrations and stability of an axially moving beam with time-dependent velocity. Int. J. Non-Linear Mech. 36, 107-115 (2001)

26. Pelicano, F., Vestroni, F.: Non-linear dynamics and bifurcations of an axially moving beam. ASME J. Vib. Acoust. 122, 21$30(2000)$

27. Pelicano, F., Fregolent, A., Bertuzzi, A., Vestroni, F.: Primary and parametric non-linear resonances of a power transmission belt. J. Sound Vib. 244, 669-684 (2001)

28. Lee, U., Oh, H.: Dynamics of an axially moving viscoelastic beam subject to axial tension. Int. J. Solids Struct. 42, 23812398 (2005)

29. Chen, L.Q., Yang, X.D.: Transverse nonlinear dynamics of axially accelerating viscoelastic beams based on 4-term Galerkin truncation. Chaos Solitons Fractals 27, 748-757 (2006)

30. Marnowski, K., Kapitaniak, T.: Zener internal damping in modeling of axially moving viscoelastic beam with time-dependent tension. Int. J. Non-Linear Mech. 42, 118-131 (2007)

31. Hou, Z.C., Zu, J.W.: Non-linear free oscillations of a moving viscoelastic belt. Mech. Mach. Theory 37, $925-940$ (2002)

32. Koivurova, H.: The numerical study of the nonlinear dynamics of a light, axially moving string. J. Sound Vib. 320, 373385 (2009)

33. Cooper, J.: Asymptotic behavior for the vibrating string with a moving boundary. J. Math. Anal. Appl. 174, 67-87 (1993) 